\title{
A DIAGONAL RECURRENT NEURAL NETWORK BASED FES SYSTEM FOR THE KNEE JOINT POSITION CONTROL
}

\author{
Jer-Junn Luh, Gwo-Ching Chang, Jin-Shin Lai*, Cheng-Kung Cheng ${ }^{+}$, Te-Son Kuo, and \\ Jau-Fa Lee
Department of Electrical Engineering, National Taiwan University Department of Physical Medicine and Rehabilitation, National Taiwan University ${ }^{*}$ Institute of Biomedical Engineering, National Yang Ming University ${ }^{+}$ Taipei, Taiwan, R.O.C. \\ ABSTRACT \\ METHODS
}

A diagonal recurrent neural network (DRNN) based functional electrical stimulation (FES) system was designed to control the knee joint to move in accordance with the desired trajectory of movement through stimulation of quadriceps muscle. This system, which consisted of a DRNN controller and a DRNN identifier, could learn the non-linearity of the plant (knee joint) and control it both in on-line condition. The knee joint angle was controlled with only small deviations along the desired trajectory with the aid of neural controller.

\section{INTRODUCTION}

Functional electrical stimulation (FES) is a promising technique in controlling electrical stimulus to the intact peripheral nerve in attempt to generate motion. FES was shown to generate the motion functions of upper limbs in quadriplegic patients and the motions of lower limbs in paraplegic patients. However, there were many obstacles in stimulating paralyzed neuro-muscular system, such as rapid fatigue, severe spasticity, time-varying properties, nonlinear length-tension, and nonlinear force-velocity of paralyzed muscles [1]. Many control strategies have been developed to provide enhanced the reproducibility of muscle responses. However, those control strategies did not satisfy the desire of FES. In previous study, we proved that an artificial neural network is a better alternative for the modeling and control of complex nonlinear systems because it can map arbitrarily complicated nonlinear input/output relationships from a given training data set [2]. One major limitation of previous studies was that the neural network needed some given data to 'learning' the properties of the plant in off-line condition. In this studies we used a DRNN to overcome this disadvantage.
The experimental set-up of this study was same as [2]. Briefly, the knee extensors (quadriceps muscle group) were stimulated by a pair of surface carbon-filled silicone electrodes $(40 \times 80 \mathrm{~mm})$. A PC with an Intel $80486 \mathrm{CPU}$ was used to control the electric. Two able-bodied and one paraplegic male subject participated in this study. The knee joint angle was measured via a potentiometer.

The block diagram of the control system was illustrated in Fig. 1 [3]. This control system contained a DRNN controller and a DRNN identifier. The plant to be controlled represented the quadriceps-lower leg system. Given a desired reference trajectory of movement, the neural controller can calculate the required stimulus activation of the quadriceps muscle. In the same time, the DRNN identifier was used to learn the properties of the plant.

The DRNN is a modified model of the fully connected recurrent neural network with one hidden layer, and the hidden layer is comprised of self-recurrent neuron. In this study, the input of neural network was composed of both the plant input and output information. This network had two neurons in the input layer and one neuron in the output layer. The number of hidden neurons for the network was determined based on the learning performance. The activation function of the hidden-layer neurons was a differentiable nonlinear sigmoid function. In the output layer, the neuron activation function was linear.

The network parameter to be determined was the number of hidden neurons. In determining the number of hidden neurons, the goal was to find the fewest possible hidden neurons. The improvement in learning was evaluated by the training error (sum of square error, SSE).

The DRNN training algorithm is same as [3]. Briefly, this algorithm is a dynamic error 
backpropagation (DBP) method which can 'catch' the information contained in the plant on-line. It should be noted that $\theta_{\mathrm{u}}(\mathrm{t})$ represents the sensitivity of the plant as below:

$\theta_{\mathcal{u}}(t) \equiv \frac{\partial \theta(t)}{\partial I(t)} \ldots \ldots \ldots \ldots \ldots \ldots \ldots \ldots \ldots \ldots \ldots \ldots \ldots \ldots$

To demonstrate the tracking abilicy of the control system, a $0.5 \mathrm{~Hz}$ and a $1 \mathrm{~Hz}$ sinusoidal sequences were considered as reference trajectories of knee joint movement. The root-mean-square (rms.) error between the desired and actual trajectory was used to quantify the quality of the tracking performance.

\section{RESULTS}

Our preliminary results from the control of knee joint indicated that the trained neural network controller was capable of roughly controlling the knee joint to follow the desired knee joint position with small tracking errors. The DRNN could trace the desire joint angle within 2 seconds.

\section{DISCUSSION}

This study has presented a DRNN based FES system design in controlling knee joint position during quadriceps stimulation. The performance of the knee joint position control based on the DRNN is similar as our previous study [2].

In practical, the training signals must be obtained by experiments for each individual. In this study, the training data set was constructed by considering sufficient excitation of the system for a range of movements. In previous we used a lowpass filtered random sequence with uniform distribution was used as input signals to stimulate quadriceps to reveal the dynamical characteristics of the FES-induced movement of knee. The new DRNN identifier can also learn the characteristics that mentioned al ove.

The neural control strategy is valid for inding the nonlinear system that the mathematical model is not available. Howe ver, it may be difficult to understand how it works. There is no simple reiationship between the learned weights of the network and the parameters of the plant. The performance of neural controller is limited by several factors: (i) the accuracy with which the plant can be modeled, (ii) the degree to which the plant can be approximated by a neural network, and (iii) the variations with time and input of the plant dynamics. Compared with our previous work, we used a DRNN based FES system to overcome the dynamics. Therefore, no PID controller was needed in this system. Besides, the neural controller with an on-line adaptation algorithm for adjusting the neural connection weights can promote the control performance of the feedforward neuro-controller in our previous study.

The knee joint position control is a valnable insight into the control of FES-induced paraplegic standing and walking. This control system has demonstrated the following advantages: (1) it uses only input/output data for its operation and does not require a system model, and (2) it can generalize the learned muscle stimulation patterns to new movements.

Future work will include the development of controllers for generating standing and walking functions with the same DRNN architecture.

\section{REFERENCES}

[ 1] H. J. Chizeck, R. Kobetic, E. B. Marsolais, J. J. Abbas, I. H. Donner, and E. Simon, "Control of functional neuromuscular stimulation system for standing and locomotion in paraplegics," Proc. IEEE, vol. 76, no. 9, pp. 1155-1165, 1988.

[2] G-C Chang, J-J Luh, G-D Liao, J-S Lai, C-K Cheng, B-L Kuo, and T-S Kuo, "A neuro-control system for the knee joint position control with quadriceps stimulation," IEEE Trans. Rehabil. Eng. vol. 5, no. 1, pp.2-11, 1997.

[3] C-C Ku and K. Y. Lee, "Diagonal recurrent networks for dynamic systems control," IEEE Trans. Neural Networks. vol. 6, no. 1, pp.144-156, 1995.

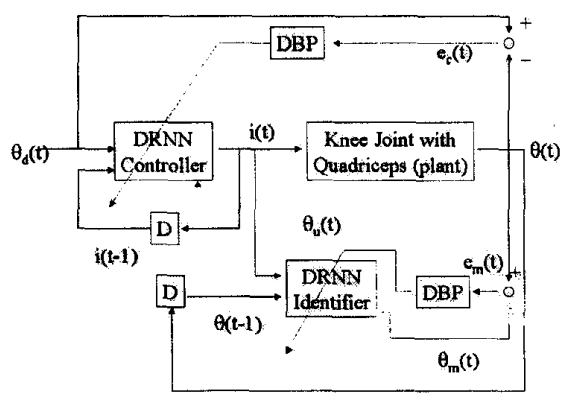

Fig. 1. Block diagram of DRNN based FES system. 